\title{
Herpes Zoster Accompanying Odontogenic Inflammation: A Case Report with Literature Review
}

\author{
Soyeon Lee, Minsik Kim, Jong-Ki Huh, Jae-Young Kim \\ Department of Oral and Maxillofacial Surgery, Gangnam Severance Hospital, Yonsei University College of Dentistry, Seoul, Korea
}

Received September 8, 2020

Revised December 21, 2020

Accepted December 21, 2020

Correspondence to:

Jae-Young Kim

Department of Oral and Maxillofacial

Surgery, Gangnam Severance Hospital,

Yonsei University College of Dentistry, 211

Eonju-ro, Gangnam-gu, Seoul 06273, Korea

Tel: +82-2-2019-4560

Fax: +82-2-3463-4052

E-mail: kjy810927@yuhs.ac

https://orcid.org/0000-0002-9423-438X

This research did not receive any specific grant from funding agencies in the public, commercial, or not-for-profit sectors.
Herpes zoster is caused by reactivation and multiplication of a latent varicella-zoster virus infection. Reactivation can frequently occur in older adults and immunosuppressed individuals. It is hypothesized that this is related to an aging society and a corresponding increase in the number of people with underlying chronic diseases, such as cancer and diabetes, that lower immunity. Clinically, the patient complains of pain, and a vesicular rash presents on one side of the face up to the midline in the dermatomes associated with the affected ganglion. Herpes zoster of the oral mucosa is rare. When oral lesions do occur, they are most often concurrent with pathognomonic unilateral linear vesicular skin lesions, facilitating both clinical diagnosis and management of the condition. Cases limited to the oral mucous membrane alone are most unusual. Treatment includes antiviral agents and analgesics for pain control. Antivirals should be administered within 72 hours of onset. Early diagnosis and treatment are important to avoid complications, such as postherpetic neuralgia. The present case report describes the adequate management of a patient diagnosed with shingles which affected the right side of the face and oral cavity. In addition, a literature review is presented.

Key Words: Chikenpox; DNA virus infections; Herpes zoster; Inflammation

\section{INTRODUCTION}

Herpes zoster is a viral DNA infection that presents unilaterally as a vesicular rash in the dermatomes corresponding to the affected ganglion [1]. Herpes zoster, clinically known as shingles, is caused by the chickenpox virus, which typically affects children between 5 and 9 years old, lurks in the sensory ganglia, and then reactivates in adults with reduced immune function [2]. Herpes zoster is highly prevalent; approximately $20 \%$ of the world's population will suffer a recurrence of this condition more than once in a lifetime [3].

According to the National Health Insurance Corporation in Korea, domestic cases of shingles have increased by $12 \%$ over five years, escalating from approximately 640,000 in
2014 to 720,000 in 2018. The incidence of shingles in the Republic of Korea has been steadily increasing by 3\% each year for the past five years [4].

Herpes zoster infection of the oral mucosa is relatively rare. Most infections affect dermatomes T3 to L2 [5]. However, an estimated 13\% of patients present with infections involving any of the three branches of the trigeminal nerve [5]. Intraorally, mucosal hyperpigmentation is evident, and the patient complains of a burning and stinging sensation of the skin and mouth [5]. Occasionally, patients complain of toothache or edema [6,7].

Herein, we report a patient who presented with blisters and vesicles affecting the right half of the face and oral mucosa, following several days of toothache, initially misdiagnosed as apical periodontitis. 


\section{CASE REPORT}

This study was approved by the institutional review board of Gangnam Severance Hospital (Registration No. \#3-2020-0009).

A 47-year-old male patient visited the Department of Oral and Maxillofacial Surgery at Gangnam Severance Hospital. His chief complaint was persistent right facial swelling for three days with newly appearing blisters around right lower lip on the previous night. He had visited a private clinic three days prior, complaining of toothache and facial swelling. Intraoral incision and drainage were performed due to suspected diagnosis of pericoronitis or apical periodontitis. However, the patient experienced no improvement in his condition. Consequently, the patient was referred to the Gangnam Severance Hospital. He had no underlying disease but did report a smoking habit.

At the first visit, a partially erupted mandibular right third molar with sensitivity to percussion and an increased mobility was observed. The surrounding gingiva was erythematous, edematous, and fluctuant, suggesting pericoronitis. Maximum mouth opening was $33 \mathrm{~mm}$. The fluctuant swelling was aspirated. However, no remarkable purulent discharge was apparent. Multiple whitish lesions affecting the right aspect of the hard palate and the right buccal mucosa were evident. Erythematous areas extending to the soft palate and including the retromolar pad were also apparent (Fig. 1A, B). Externally and unilaterally, multiple crops of coalescing vesicles along with a few shallow ulcers were present on the right side of the face and around the right oral commissure causing localized heatness (Fig. 1C, D). The facial skin was tender, soft in consistency, and had a rough surface texture. The blisters appeared to radiate from the mouth towards the cheeks and ears. The patient was referred to the Department of Dermatology with a suspected diagnosis of herpes zoster. An oral antiviral agent (famciclovir; Yuhan Co., Ltd., Seoul, Korea) was administered at $250 \mathrm{mg}$ three times daily for seven days, and an antibacterial ointment (mupirocin; JW Shinyak Co., Ltd., Seoul, Korea) was prescribed by the dermatologist. In addition, an antibiotic (cefdinir; Jeil Pharmaceutical Co., Ltd., Yongin, Korea) and analgesic (ibuprofen arginine; Ildong Pharmaceutical Co., Ltd., Yongin, Korea) was prescribed for five days to prevent secondary infection and pain.

The following day (i.e., the second visit), the extraoral blisters and intraoral erythematous whitish lesions appeared to have worsened (Fig. 2). On panoramic x-ray, a periapical radiolucency associated with the mandibular right third
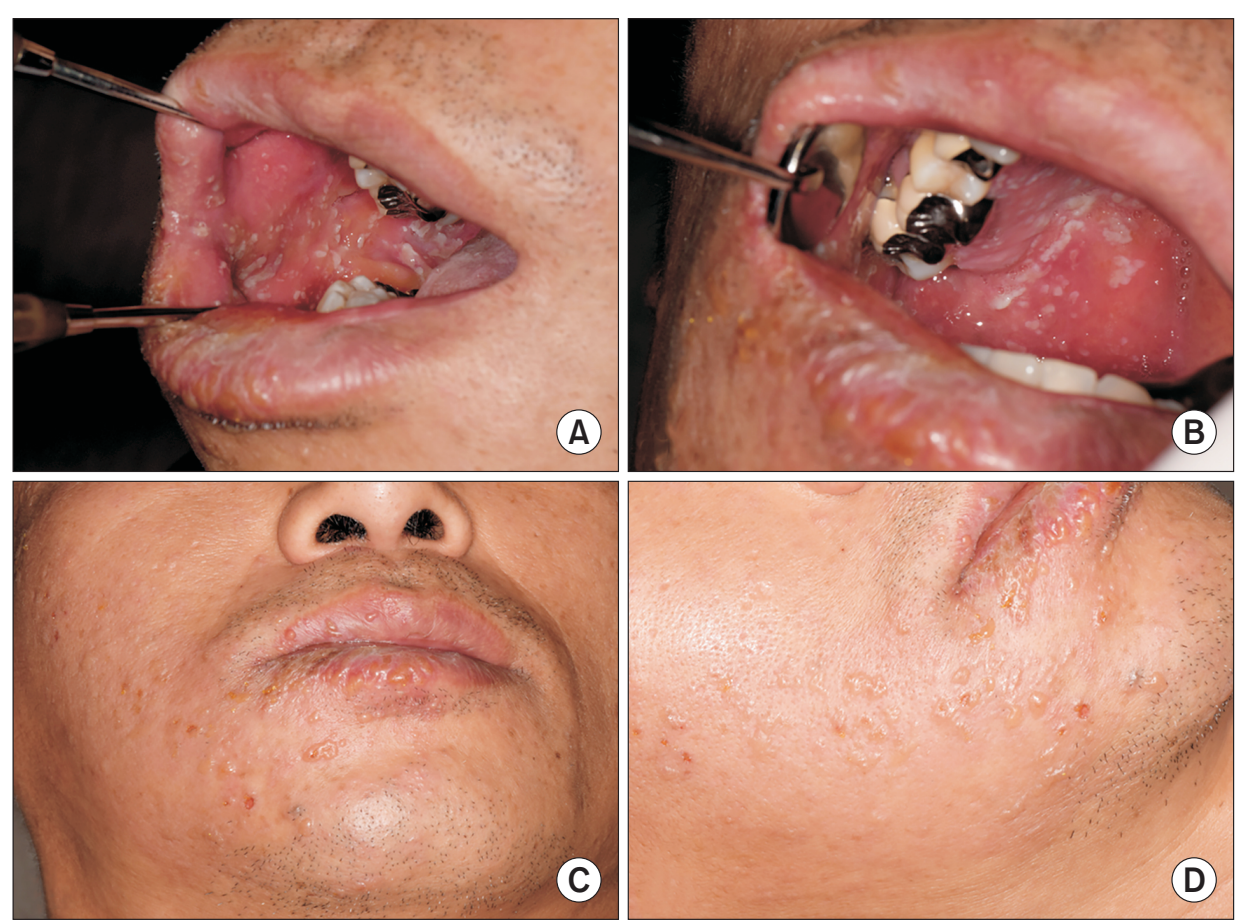

Fig. 1. First visit. (A, B) Erythematous areas with whitish lesions extending from the right mandibular third molar and retromolar pad to the soft palate. (C, D) Extraoral blisters around the right oral commissure and chin show the distribution of the involved nerve. 

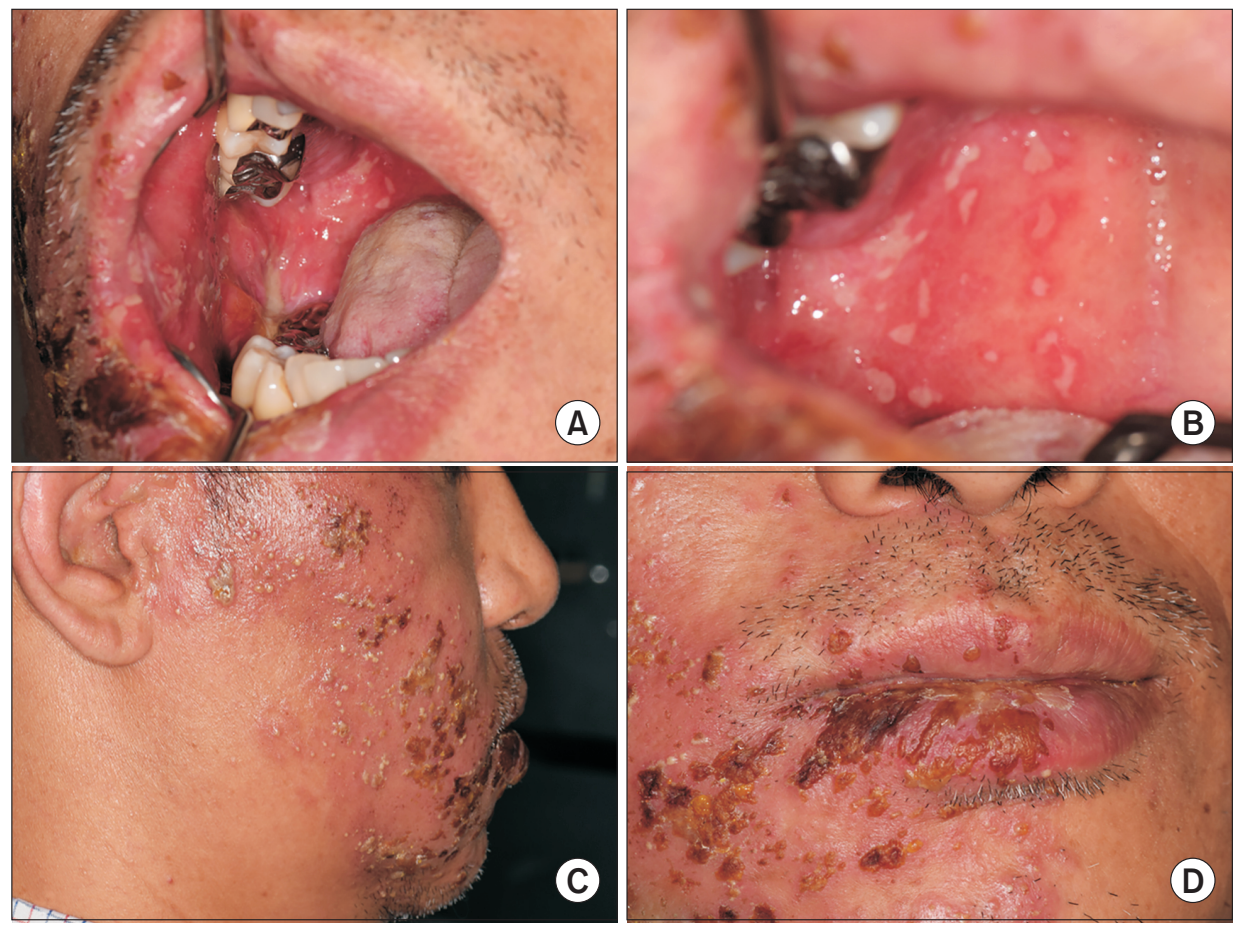

Fig. 2. Second visit. (A-D) Extraoral blisters and intraoral erythematous areas with whitish lesions appear to have worsened.

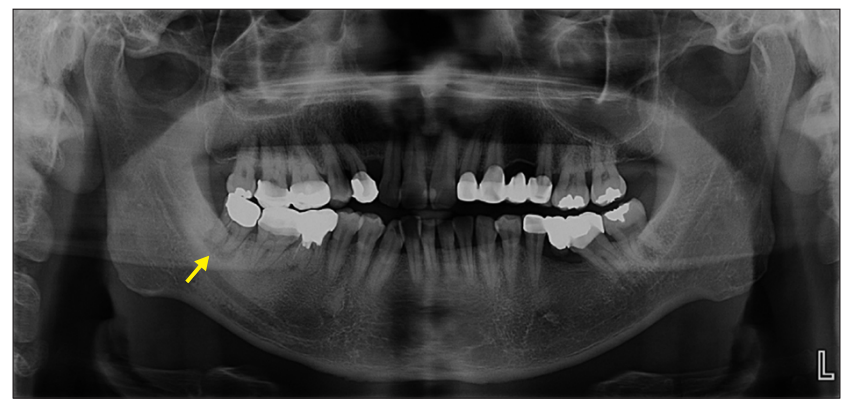

Fig. 3. Panoramic radiograph showing an apical radiolucency associated with the mandibular right third molar.

molar was apparent (Fig. 3). The fluctuant area was aspirated again. Once again, no purulent discharge was noted. White blood cell count $(4,880 / \mu \mathrm{L}$, reference range: $4,000-$ $10,800 / \mu \mathrm{L}$ ) and C-reactive protein levels $(3.8 \mathrm{mg} / \mathrm{L}$, reference range: 0.1-6.0 $\mathrm{mg} / \mathrm{L}$ ) were within normal range.

The next day (i.e., the third visit), facial pain and toothache had subsided from the previous day, but incision and drainage were performed as the intraoral and extraoral swelling had clinically deteriorated. However, once again, no suppuration was observed. Still, maximum mouth opening was $33 \mathrm{~mm}$.

Two days later (i.e., the fourth visit), the swelling surrounding the third molar had subsided, and mouth opening capacity had increased to $45 \mathrm{~mm}$. However, there was still extraoral swelling. The extraoral swelling subsided two days later. Once the oral lesions had resolved, five weeks after the first visit to the hospital, the maxillary and mandibular right third molars were extracted. The extraction sockets healed without complications. Four weeks after the first visit, as a postherpetic neuralgia developed, and the patient was referred to the Department of Family Medicine for further care.

\section{DISCUSSION}

Herpes zoster (HZ) infection of the cranial nerves accounts for $20 \%$ of the total cases and most commonly affects the trigeminal nerve. $\mathrm{HZ}$ infection of the trigeminal nerve is a disease that falls within the diagnostic scope of all dentists and dental specialists. The clinical features of HZ can be grouped into three phases: (1) prodromal, (2) acute, and (3) chronic. Prodromal symptoms include neuropathic pain, headache, malaise, and disrupted sleep [1]. During the prodromal stage in particular, the only presenting symptoms may be similar to pulpitis [7]. Approximately $10 \%$ of affected individuals will exhibit no prodromal pain. Within a few days, epithelial lesions appear, presenting as 
multiple papules affecting one-half of the face, increase rapidly in size, develop into fluid-filled vesicles within 1224 hours, and rupture to form ulcers with a fluid discharge [3]. Initially, the lesions in the affected dermatome are clustered at a few sites. As the disease progresses, the vesicles often coalesce into larger, fluid-filled lesions. Finally, the blisters crust over and disappear [3]. On occasion, a recurrence in the absence of vesiculation of the skin or mucosa may occur.

Oral lesions occur when the trigeminal nerve is involved and may affect the attached or movable mucosa. Lesions present as fluid-filled vesicles and erythematous papules. These lesions often extend to the midline and occur in conjunction with a facial rash [8].

Zoster-associated complications include neurologic components such as Guillain-Barre syndrome, encephalitis, myelitis, Ramsey-Hunt Syndrome, and Horner's syndrome. Less well recognized maxillofacial complications include developmental anomalies, such as irregular short roots and missing teeth, facial scarring, periodontitis, calcified and devitalized pulps, periapical lesions, alveolar necrosis, tooth exfoliation, and resorption of roots $[9,10]$.

Postherpetic neuralgia can sometimes occur, and depending on the branch of the trigeminal nerve that is involved, it affects the upper eyelid, forehead, and scalp (V1); midface and upper lip (V2); or lower face and lower lips (V3) [5]. In the present case, the maxillary (V2) and mandibular (V3) divisions were involved. Postherpetic neuralgia is a neuropathy resulting from peripheral and central nervous system injury and altered central nervous system processing. The recommended therapy should include patient isolation; local management of the skin lesions; pain control; and the extent, duration, and severity of the disease should be limited through the prescription of antiviral medications [5].

In the present case, excluding swelling, the general characteristics of oral zoster were seen and clinically diagnosed as HZ. Nevertheless, inflammation could not be excluded because there was also gingivitis swelling in which the patient's third molar exhibited apical radiolucency. There was no clear pus discharge, but it is possible that after antibiotics treatment, inflammation was controlled and swelling and opening disorders were reduced. Also, erythema during the prodromal stage can mimic pericoronitis. Likewise, the skin lesions associated with erythema multiforme can mimic those of herpes zoster. In other words, the signs and symptoms of a herpes zoster infection of the mandibular branch of the trigeminal nerve can be misdiagnosed [7]. According to research data, blisters that develop within 12 to 24 hours after the early prodromes of herpes infection are experienced in the skin overlying the nerve distribution, causing burning, tingling, itching, boring, prickly, or knifelike sensations. Unilateral lesions, typically involving the dermatomes, and an absence of target lesions may help to exclude a diagnosis of erythema multiforme.

The patient initially experienced pain around the mandibular right third molar. Therefore, antibiotics and analgesics were prescribed to control pain and prevent a secondary infection in the future. Oral famciclovir (250 mg three times daily for seven days), and an antibacterial ointment (mupirocin) were prescribed by a dermatologist. Symptoms improved following administration of the antiviral agent.

In conclusion, orofacial pain due to herpes at the prodromal stage, before the development of blisters, can mimic or accompany symptomatic apical periodontitis or pericoronitis. Therefore, dentists should have a thorough knowledge of the clinical characteristics of $\mathrm{HZ}$, the treatment, and possible complications thereof. An appropriate diagnosis must be established prior to any invasive procedure, such as endodontic therapy or extraction.

\section{CONFLICT OF INTEREST}

No potential conflict of interest relevant to this article was reported.

\section{ORCID}

\author{
Soyeon Lee \\ https://orcid.org/0000-0001-9558-734X \\ Minsik Kim \\ https://orcid.org/0000-0002-0275-7299 \\ Jong-Ki Huh \\ https://orcid.org/0000-0002-7381-3972 \\ Jae-Young Kim \\ https://orcid.org/0000-0002-9423-438X
}




\section{REFERENCES}

1. Tomar N, Lalawat S, Soman BP, Das D, Malusare P. Vague and versatile - herpes zoster, 2 case reports and review. EC Dent Sci 2019;18:2107-2115.

2. Eisenberg E. Intraoral isolated herpes zoster. Oral Surg Oral Med Oral Pathol 1978;45:214-219.

3. Park YM. Comprehensive review and update on herpes zoster. J Korean Med Assoc 2018;61:116-122.

4. National Health Insurance Service. Women in their 50s and older received the most treatment for herpes zoster [Internet]. Seoul: National Health Insurance Service; c2019 [cited 2019 Aug 1]. Available from: https://www.nhis.or.kr/nhis/together/ wbhaea $01600 \mathrm{m01}$. do mode $=$ view $\&$ articleNo $=131060 \&$ article . offset $=450 \&$ articleLimit $=10$.

5. Raj SS, Verma P, Mahajan P, Puri A. Herpes zoster infection of the face: a case report with review of literature. J Indian Acad
Oral Med Radiol 2017;29:159-161.

6. Keskinruzgar A, Demirkol M, Ege B, Aras MH, Ay S. Rare involvement of herpes zoster in the mandibular branch of the trigeminal nerve: a case report and review of the literature. Quintessence Int 2015;46:163-170.

7. Yang SH, Jung DH, Lee HD, Lee Y, Chang HS, Min KS. Clinical diagnosis of herpes zoster presenting as odontogenic pain. J Korean Acad Conserv Dent 2008;33:452-456.

8. Wright WE, Davis ML, Geffen DB, Martin SE, Nelson MJ, Straus SE. Alveolar bone necrosis and tooth loss: a rare complication associated with herpes zoster infection of the fifth cranial nerve. Oral Surg Oral Med Oral Pathol 1983;56:39-46.

9. Jain MK, Manjunath KS, Jagadish SN. Unusual oral complications of herpes zoster infection: report of a case and review of literature. Oral Surg Oral Med Oral Pathol Oral Radiol Endod 2010;110:e37-e41.

10. Rauckhorst AJ, Baumgartner JC. Zebra. XIX. Part 2. Oral herpes zoster. J Endod 2000;26:469-471. 